\title{
Condensation energy in strongly coupled superconductors.
}

\author{
Robert Haslinger ${ }^{1}$, Andrey V. Chubukov ${ }^{2}$ \\ 1 Los Alamos National Laboratory, Los Alamos, NM 87545 and \\ 2 Department of Physics, University of Wisconsin, Madison, WI 53706
}

(Dated: November 2, 2018)

\begin{abstract}
We consider the condensation energy, $E_{c}$, in superconductors where the pairing is electronic in origin and is mediated by a collective bosonic mode. We use magnetically-mediated superconductivity as an example, and show that for spin-fermion couplings $\lambda \sim 1$, the physics is qualitatively different from the BCS theory as the condensation energy results from the feedback on spin excitations, while the electronic contribution to $E_{c}$ is positive due to an "undressing" feedback on the fermions. The same feedback effect accounts for the gain of the the kinetic energy at strong couplings.
\end{abstract}

PACS numbers: 74.25.-q, 74.72.-h, 61.12.-q

Undertanding the origin of the condensation energy is an important step towards identifying the mechanism of high temperature superconductivity in the cuprates. In a BCS superconductor, the condensation energy - the energy gained upon entering the superconducting state, is $E_{c}^{B C S}=-N_{f} \Delta^{2} / 2$, where $\Delta$ is the superconducting gap and $N_{f}$ is the fermionic density of states. [1] This decrease in the total energy upon pairing results from a fine competition between an increased kinetic energy and a decreased potential energy, both of which are much larger than $E_{c}$. For optimally doped $Y B C O$ with $N_{f} \sim$ $10^{-3} \mathrm{meV}^{-1}$, and $\Delta \sim 30 \mathrm{meV}$, the BCS formula yields $E_{c} \sim 5 K$ in reasonable agreement with $E_{c} \approx 0.12 k_{B} T_{c} \sim$ $10 K$ that Loram et al [2] extracted from specific heat measurements. However, at smaller dopings, $\Delta$ increases while the measured $E_{c}$ decreases, in clear disagreement with the BCS theory.

In this paper, we present a computation of the condensation energy for a strongly coupled superconductor. We argue that at strong coupling, the relation between $E_{c}$ and $\Delta$ is different from BCS theory, and is consistent with experimental trends in the underdoped cuprates. We show that the agreement with the BSC theory at optimal doping is largely coincidental as at this doping range, strong coupling effects are already dominant.

Some earlier works have already suggested that the gain in the condensation energy in the cuprates may be due to a non BCS physics. Scalapino and White [3] conjectured that at strong coupling, the dominant contribution to the condensation energy comes from a feedback effect on the magnetic excitations of the system. On the other hand, Norman et al argued [4] that the condensation energy likely has an electronic origin, and is driven by a gain in the kinetic energy which at strong coupling is negative (in contrast to BCS theory) because of a strong "undressing" of fermions which bear a greater resemblance to free particles in the superconducting state than they do in the normal state. Similar ideas were expressed by Hirsh and Marsiglio [5]. A different idea, related to the lowering of the Coulomb energy in the superconducting state has been proposed by Leggett [6].

In this communication we argue that these apparently disparate viewpoints are in fact consistent with each other, and describe the same strong coupling physics. In the superconducting state, spin decay into fermions is forbidden at energies smaller than $2 \Delta$. This simultaneously gives rise to two effects. First, the spin propagator develops an excitonic (resonance) peak at $\omega_{\text {res }}<2 \Delta$. The energy released by the creation of an exciton results in a gain in the magnetic part of the condensation energy. Secondly, the fermions cannot decay until their frequency exceeds $\Delta+\omega_{\text {res }}$ (this is the magnetic ana$\log$ of the Holstein effect). The elimination of fermionic scattering at low frequencies implies that the fermionic self-energy $\Sigma(\omega)$ in the superconducting state is reduced compared to that in the normal state. This effect lowers the kinetic energy (see below) and at strong coupling overcomes the effect of particle-hole mixing that increases $E_{k i n}$ in a BCS superconductor.

The above reasoning is quite general and can be applied to various superconducting mechanisms. A more subtle issue is to explain the reduction of $E_{c}$ in the underdoped regime, and why $E_{c} \sim 10 K$ at optimal doping despite the fact that by all accounts, strong coupling effects are already large. Below we present an explicit computation of $E_{c}$ assuming that the pairing is due to spin-fluctuation exchange We show that our theoretical $E_{c}$ agrees with the data both in magnitude and doping dependence. We view this agreement as support for the spin-fluctuation scenario for the cuprates.

The condensation energy $E_{c}$ is the difference between the grand free energies in the superconducting and normal states [9] $E_{c}=\Omega_{s}-\Omega_{n}$. We obtained $E_{c}$ by modifying the Eliashberg formula for phonon superconductors [7, 8] to the spin case. This approach implies that $E_{c}$ is dominated by momenta near the hot spots where the momentum dependence of the $d$-wave gap can be neglected except for the sign change between "hot" regions. We will discuss the validity of the Eliashberg theory for the spin case after we present the results. In the Eliashberg theory, $E_{c}$ can be represented as the sum of two parts $E_{c}=\delta \Omega_{e l}+\delta \Omega_{\text {spin }}$, where

$$
\begin{aligned}
\Omega_{e l} & =-T \sum_{m} \int \frac{d^{2} k}{(2 \pi)^{2}}\left(\log \left[\epsilon_{\mathbf{k}}^{2}+\widetilde{\Sigma}_{\omega_{m}}^{2}+\Phi_{\omega_{m}}^{2}\right]\right. \\
& \left.-i \Sigma_{\omega_{m}} G_{\omega_{m}}(k)+i \Phi_{\omega_{m}} F_{\omega_{m}}(k)\right)
\end{aligned}
$$




$$
\begin{aligned}
\Omega_{\text {spin }} & =\frac{3}{2} T \sum_{m} \int \frac{d^{2} q}{(2 \pi)^{2}}\left[\log \left(\chi^{-1}\left(q, \omega_{m}\right)\right)\right. \\
& \left.+\Pi_{\omega_{m}} \chi\left(q, \omega_{m}\right) / \chi(Q, 0)\right] .
\end{aligned}
$$

Here $\left.G_{(} \omega_{m}\right)$ and $F\left(\omega_{m}\right)$ are the real and anomalous Greens functions given by $G_{\omega_{m}}(k)=-\left(\epsilon_{\mathbf{k}}+i \widetilde{\Sigma}_{\omega_{m}}\right) /\left(\epsilon_{\mathbf{k}}^{2}+\right.$ $\left.\widetilde{\Sigma}_{\omega_{m}}^{2}+\Phi_{\omega_{m}}^{2}\right)$ and $F_{\omega_{m}}(k)=i \Phi\left(\omega_{m}\right) /\left(\epsilon_{\mathbf{k}}^{2}+\widetilde{\Sigma}_{\omega_{m}}^{2}+\Phi_{\omega_{m}}^{2}\right)$, where $\epsilon_{\mathbf{k}}=v_{F}\left(k-k_{F}\right)$, and $\widetilde{\Sigma}_{\omega_{m}}=\omega_{m}+\Sigma_{\omega_{m}}$. The conventionally defined pairing gap $\Delta_{\omega_{m}}$ is the ratio of the anomalous vertex and the self-energy: $\Delta_{\omega_{m}}=$ $\Phi_{\omega_{m}} \omega_{m} / \tilde{\Sigma}_{\omega_{m}}$. In the second term, $\chi(\mathbf{q}, \omega)$ is the dynamical spin susceptibility related to the spin polarization operator $\Pi_{\omega_{m}}$ by $\chi\left(\mathbf{q}, \omega_{m}\right)=\chi(Q, 0) /\left(1+\xi^{2}(\mathbf{q}-\mathbf{Q})^{2}-\right.$ $\left.\Pi_{\omega_{m}}\right)$ where $\chi(Q, 0)$ is the static staggered susceptibility. The factor of 3 in $\Omega_{\text {spin }}$ is due to the spin summation.

The term $\delta \Omega_{e l}=\Omega_{e l}^{s c}-\Omega_{e l}^{n}$ accounts explicitly for the appearance of the anomalous pairing vertex $\Phi_{\omega_{n}}$, and for the feedback changes to the fermionic self-energy. The term $\delta \Omega_{\text {spin }}=\Omega_{\text {spin }}^{s c}-\Omega_{\text {spin }}^{n}$ accounts for changes to the spin propagator via the changes to the spin polarization operator $\Pi_{\omega_{m}}$. This term is almost negligible for phonon superconductors [10]. It is however important for magnetic superconductors where the bosonic mode that mediates the superconductivity is by itself affected by the pairing.

The fermionic self-energy $\Sigma_{\omega_{m}}$, the pairing vertex $\Phi_{\omega_{m}}$, and the spin polarization operator $\Pi_{\omega_{m}}$ are the solutions of three coupled Eliashberg equations [11]

$$
\begin{aligned}
\Sigma_{\omega_{m}} & =\pi \lambda T \sum_{n} \frac{\widetilde{\Sigma}_{\omega_{n}}}{\sqrt{\widetilde{\Sigma}_{\omega_{n}}^{2}+\Phi_{\omega_{n}}^{2}}} \frac{1}{\left(1-\Pi_{\omega_{m}-\omega_{n}}\right)^{1 / 2}}, \\
\Phi_{\omega_{m}} & =\lambda \pi T \sum_{n} \frac{\Phi_{\omega_{n}}}{\sqrt{\tilde{\Sigma}_{\omega_{n}}^{2}+\Phi_{\omega_{n}}^{2}}} \frac{1}{\left(1-\Pi_{\omega_{m}-\omega_{n}}\right)^{1 / 2}}, \\
\Pi_{\omega_{m}} & =\frac{4 \lambda^{2}}{\bar{\omega}} \pi T \sum_{n}[-1 \\
& \left.+\frac{\widetilde{\Sigma}_{\omega_{n}} \widetilde{\Sigma}_{\omega_{n}+\omega_{m}}+\Phi_{\omega_{n}} \Phi_{\omega_{n}+\omega_{m}}}{\sqrt{\widetilde{\Sigma}_{\omega_{n}}^{2}+\Phi_{\omega_{n}}^{2}} \sqrt{\widetilde{\Sigma}_{\omega_{n}+\omega_{m}}^{2}+\Phi_{\omega_{n}+\omega_{m}}^{2}}}\right] .
\end{aligned}
$$

Eqs. (2) contain only two inputs: the overall energy scale $\bar{\omega}$ that is set by the spin-fermion interaction, and the dimensionless spin-fermion coupling $\lambda \propto \xi$ that diverges as the system approaches the antiferromagnetic instability. Physically, the energy scale $\bar{\omega}$ is the ultimate upper cutoff for the strong coupling behavior $\left(\Sigma_{\omega_{m}}<\omega_{m}\right.$ for $\omega_{m}>\bar{\omega}_{m}$ ), while dimensionless $\lambda$ can be represented as the ratio $(2 \lambda)^{2}=\bar{\omega} / \omega_{s f}$ of $\bar{\omega}$ and another typical scale $\omega_{s f}$ that sets the upper boundary of the Fermi-liquid behavior in the normal state.

The evolution of the condensation energy with $\lambda$ can be understood as follows. At small $\lambda$, i.e., at weak coupling, $\bar{\omega}<\omega_{s f}$, and the system behaves as a conventional Fermi liquid. In this limit, the pairing potential is static, i.e., the condensation energy is entirely electronic and BCS like. At strong couplings, i.e. $\lambda \geq 1, \bar{\omega}>\omega_{s f}$, there is a frequency range between $\omega_{s f}$ and $\bar{\omega}$ within which the system behaves as a non Fermi liquid. Previous studies demonstrated 111] that in this regime both the onset of the pairing instability and the magnitude of $\Delta_{\omega}$ at $T=0$ are determined by $\bar{\omega}$, and hence the condensation energy results predominantly from fermions located in the nonFermi liquid frequency range. For these fermions, retardation effects not included in the BCS theory become dominant, hence one expects strong deviations from the BCS form of $E_{c}$. Comparison to experiment [4, 11] yields $\lambda \sim 1.5-2$ at optimal doping. This doping already corresponds to a strong coupling $\left(\bar{\omega} \sim(10-16) \omega_{s f}\right)$.

As $\Sigma$ and $\Phi$ depend only on $\omega$, the momentum integration in Eqs. (1) can be performed explicitly and yields

$$
\begin{aligned}
\delta \Omega_{e l}= & -N_{f} \pi T \sum_{m}\left(\sqrt{\widetilde{\Sigma}_{s, \omega_{m}}^{2}+\Phi_{\omega_{m}}^{2}}-\left|\widetilde{\Sigma}_{n, \omega_{m}}\right|\right. \\
+ & \left.\left|\omega_{m}\right| \frac{\left|\widetilde{\Sigma}_{s, \omega_{m}}\right|-\sqrt{\widetilde{\Sigma}_{s, \omega_{m}}^{2}+\Phi_{\omega_{m}}^{2}}}{\sqrt{\widetilde{\Sigma}_{s, \omega_{m}}^{2}+\Phi_{\omega_{m}}^{2}}}\right) \\
\delta \Omega_{\text {spin }}= & -N_{s} \pi T \sum_{m} \\
& \left(\tilde{\Pi}_{s, \omega_{m}}-\tilde{\Pi}_{n, \omega_{m}}+\omega_{s f} \log \frac{\omega_{s f}-\tilde{\Pi}_{s, \omega_{m}}}{\omega_{s f}-\tilde{\Pi}_{n, \omega_{m}}}\right)(4)
\end{aligned}
$$

where $\tilde{\Pi}_{\omega_{m}}=\omega_{s f} \Pi_{\omega_{m}}$. In the normal state, $\tilde{\Pi}_{\omega_{m}}=$ $-\left|\omega_{m}\right|$. We introduce $N_{f}$ via $\int d^{2} k /\left(4 \pi^{2}\right)=N_{f} \int d \epsilon_{k}$ and an analogous spin "density of states" $N_{s}=\left(8 / 3 \pi^{2}\right) \bar{\omega} / v_{F}^{2}$, where $v_{F}$ is the Fermi velocity. One can make sure that for $\lambda \geq 1$, the frequency sums in both terms yield $O\left((\bar{\omega})^{2}\right)$, hence $\delta \Omega_{e l} \sim N_{f} \bar{\omega}^{2}$, while $\delta \Omega_{\text {spin }} \sim N_{s} \bar{\omega}^{2}$.

In Fig.11 we present the results for the electronic and spin contributions to the condensation energy for different values of the coupling $\lambda$. We used previously obtained solutions of the Eliashberg equations at $T \approx 0$ in real frequencies, and related $\delta \Omega$ with $\Sigma_{\omega}, \Phi_{\omega}$ and $\Pi_{\omega}$ along the real frequency axis using the spectral representation.

There are three striking features of Fig.11. First, the electronic contribution to the condensation energy is positive. As in the BCS limit the electronic condensation energy is negative and equal to $-N_{f} \Delta^{2} / 2$, this implies that the electronic condensation energy changes sign at a rather small $\lambda \sim 0.4$, and is positive for all $\lambda \geq 1 / 2$ presented in the figure. Second, for all $\lambda$ shown, the spin part $\delta \Omega_{\text {spin }}$ is negative. Third, at large $\lambda$, the two parts of the condensation energy nearly saturate.

We next have to find a relation between $N_{s}$ and $N_{f}$. We use a trick employed by Bardeen and Stephen 10 and introduce a "mixed" self-energy $\Sigma_{n s}$ that has the normal state form, but with the spin propagator from the superconducting state. We then introduce the functional

$$
Y_{\omega_{m}}(k)=i\left(G_{n, \omega_{m}}(k) \Sigma_{n, \omega_{m}}-G_{n s, \omega_{m}}(k) \Sigma_{n s, \omega_{m}}\right)
$$

and compute $Y=T \sum_{m} \int \frac{d^{2} k}{(2 \pi)^{2}} Y_{k, \omega_{m}}$ in two ways - first, explicitly integrating over $\epsilon_{k}$, and second, reexpressing 


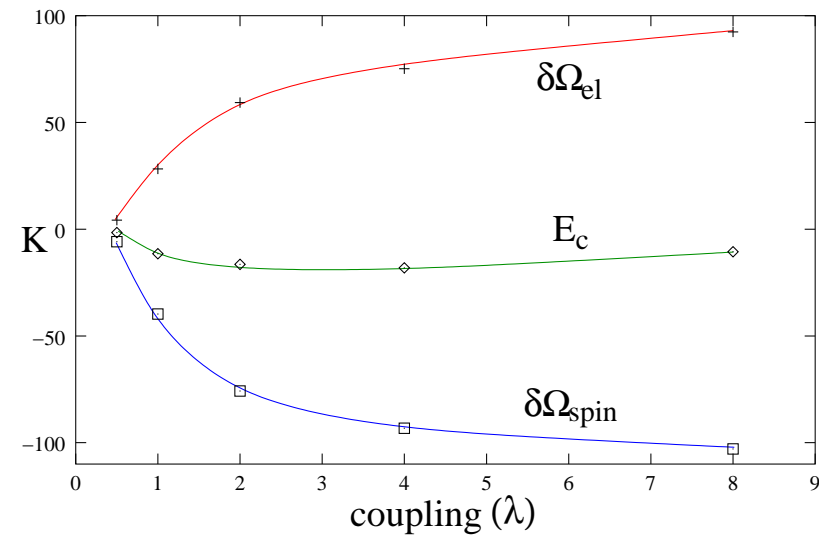

FIG. 1: Electronic $\left(\delta \Omega_{e l}\right)$, spin $\left(\delta \Omega_{\text {spin }}\right)$, and total $\left(E_{c}\right)$ condensation energy per unit cell at $T=0$ for various couplings $\lambda$. The lines are a guide for the eye. The sum of $\delta \Omega_{e l}$ and $\delta \Omega_{\text {spin }}$ produces a total condensation energy which is negative. We used $N_{f}=1 s t / \mathrm{eV}$, and $N_{s} \sim 0.17 s t / \mathrm{eV}$ as explained in the text.

the fermionic self-energy via the spin polarization operator and then integrating over the bosonic momentum. The explicit integration yields

$$
Y=\pi T N_{f} \sum_{m}\left(\left|\Sigma_{n, \omega_{m}}\right|-\left|\Sigma_{n s, \omega_{m}}\right|\right)
$$

Expressing $\Sigma$ in terms of $\Pi$, and using the fact that the polarization operator made out of $G_{n s}$ is precisely $\Pi_{n}(\omega)$ as the latter depends on the functional form of the Green's function but not on the magnitude of the self-energy [11], we find after simple algebra

$$
Y=-\pi N_{s} \sum_{m} \tilde{\Pi}_{n, \omega_{m}} \log \frac{\omega_{s f}-\tilde{\Pi}_{s, \omega_{m}}}{\omega_{s f}-\tilde{\Pi}_{n, \omega_{m}}}
$$

Comparing (5) and (6) we see that $N_{f}$ and $N_{s}$ are indeed related. We evaluated the ratio for various $\lambda$ and found that with small variations, $N_{f} / N_{s} \approx 5.9$ for all $\lambda \geq 0.5$.

In Fig. 11 we present the result for the full condensation energy $E_{c}$ using this ratio of $N_{f} / N_{s}$. To set the overall scale, we adopt a commonly used estimate $N_{f}=1 s t / e V$ 12]. We emphasize that changing $N_{f}$ will only change the overall scale and not the functional form of $E_{c}(\lambda)$. We see from the figure that the total condensation energy is negative, as it indeed should be in a superconductor, but its value is rather small due to a substantial cancellation between the spin and electronic contributions to $E_{c}$.

We also see that the condensation energy flattens at $\lambda \sim 2$, and decreases at large couplings despite the fact that the pairing gap increases monotonically with $\lambda \mid \mathbb{1 1}$. The decrease in $E_{c}$ can be understood as a reflection of the fact that as $\lambda$ increases the pairing process more and more involves the exchange of classical, on-shell bosons. Due to energy conservation, such bosons can not lead to a gain in $E_{c}$. [13. This behavior is very counterintuitive from a BCS perspective, where the condensation energy scales with $\Delta^{2}$. To further emphasize this point,

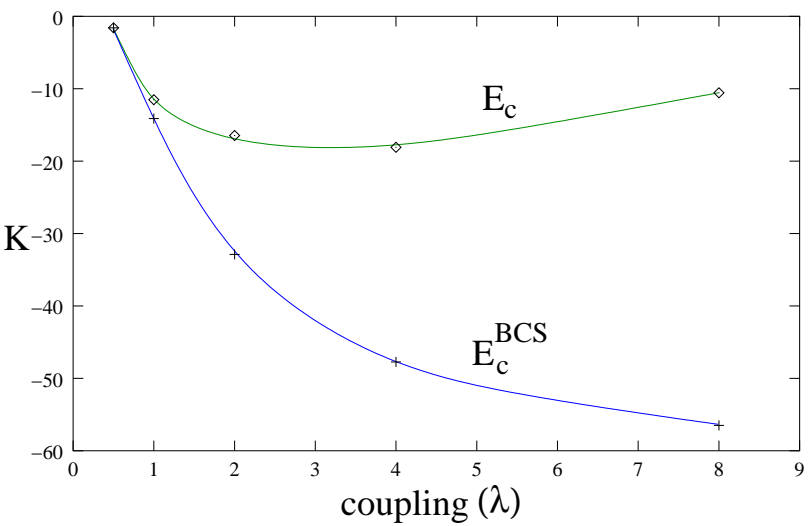

FIG. 2: Total condensation energy $E_{c}$ compared with the BCS result $E_{c}^{B C S}$ at $T=0$ for various couplings $\lambda$. We used $N_{f}=1 s t / e V$ and $N_{f} / N_{s} \sim 5.9$ as explained in the text. Observe that the BCS condensation energy increases monotonically as the coupling gets larger, while the actual condensation energy flattens at $\lambda \sim 2$ and decreases slightly at large couplings.

we plot in Fig.2 our $E_{c}$ and the BCS condensation energy $-N_{f} \Delta^{2} / 2$ with the same $\Delta$ and $N_{f}$. We clearly see that for $\lambda \geq 1$, corresponding to optimally doped and underdoped cuprates, BCS theory yields qualitatively incorrect results for $E_{c}$.

We next present the results for the change in the kinetic energy when the system enters the superconducting state. The conventionally defined kinetic-energy for an interacting fermionic system is

$$
E_{k i n}=2 T \sum_{m} \int \frac{d^{2} k}{(2 \pi)^{2}} \epsilon_{k} G_{\omega_{m}}(k)
$$

where $G_{\omega_{m}}(k)$ is the full fermionic Green's function that contains the self-energy. Integrating over momentum and subtracting the normal state result from $E_{k i n}$ in a superconductor we obtain

$$
\delta E_{k i n}=2 N_{f} \pi T \sum_{m} \sqrt{\widetilde{\Sigma}_{s, \omega_{m}}^{2}+\Phi_{\omega_{m}}^{2}}-\left|\widetilde{\Sigma}_{n, \omega_{m}}\right|
$$

In the BCS limit, $\Phi_{\omega_{n}}=\Delta, \Sigma=0, \widetilde{\Sigma}_{\omega_{n}}=\omega_{n}$ and $\delta E_{k i n}=2 N_{f} \pi T \sum_{m} \sqrt{\omega_{m}^{2}+\Delta^{2}}-\left|\omega_{m}\right|$ which is obviously positive. The results at finite $\lambda$ are presented in Fig 3. We used the same computational procedure as before. At low couplings the kinetic energy is positive, as one naively expects. At larger $\lambda$, however, the kinetic energy passes through a maximum at $\lambda \sim 2$ and then becomes negative at large $\lambda$. This implies, as we anticipated, that at strong coupling, the lowering of $E_{k i n}$ via the change in the self-energy due to the "undressing" of fermions overcomes the effect of particle-hole mixing that increases $E_{k i n}$. This behaviour is very similar to that obtained by Norman et. al. 44.

As the first term in $\delta \Omega_{e l}$ is equal to $-\delta E_{k i n} / 2$ (eqs. 4 and (8), one can indeed argue that the condensation energy at large couplings is at least partly driven by the lowering of the kinetic energy. However, a comparison 


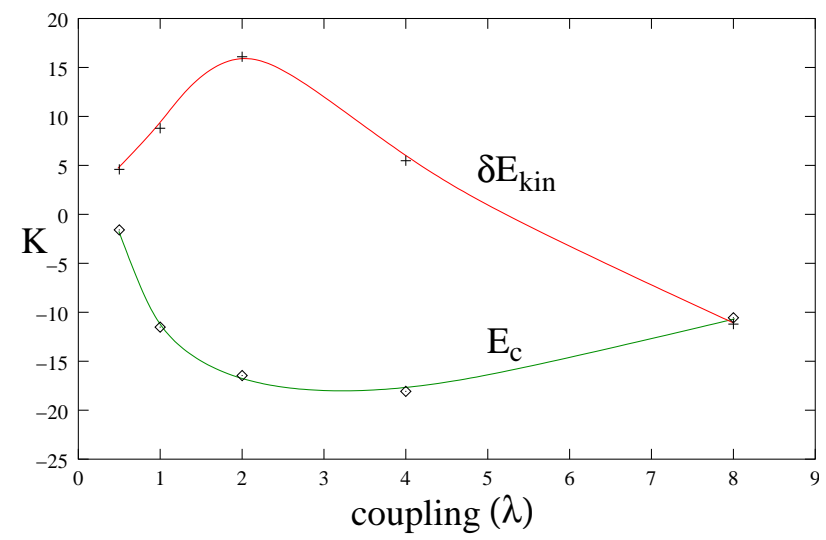

FIG. 3: Kinetic energy $\delta E_{k i n}$ compared with total condensation energy $E_{c}$ at $T=0$ for various couplings $\lambda$. The parameters are the same as in Fig. 2. The kinetic energy change is positive at low couplings, but negative at high coupling.

of Figs. 11 and 3 shows that this is just another way to interpret strong coupling effects that affect both the fermionic and bosonic propagators via mutual feedback.

We emphasize that the calculated value of $E_{c}$ is small, only $\sim 15 K$ around optimal doping. This is rather remarkable as all typical energies in the problem are much higher, i.e $\bar{\omega} \sim(2.5-3) 10^{3} K$. This small value of $E_{c}$ is partly due to small prefactors, but is also the result of substantial cancellation between the spin and electronic contributions to $E_{c}$. Loram et al [2] extracted $E_{c} \approx 0.12 k_{B} T_{c} \sim 10 K$ from the jump of the specific heat at $T_{c}$. Our result is is very close to this value.

Loram et al found that the condensation energy drops as one moves into the underdoped regime [2]. We also found a decrease in $E_{c}$ at large couplings, although not as spectacular as in Loram's experiments. This is due to the strong dispersion of the bosonic propagator assumed in the spin-fermion model. A weaker spin excitation dispersion, as suggested by neutron experiments 14, should result in a stronger reduction of $E_{c}$.

Finally, we discuss the accuracy of the Eliashberg approach and also clarify what we mean by strong coupling. As discussed previously [11, the validity of Eliashberg theory is related to the development of an "effective" Migdal theorem at strong coupling allowing vertex corrections to be neglected. As spin fluctuations become overdamped due to spin-fermion exchange they become slow compared to fermions (just as phonons are slow compared to electrons). This effective Migdal theorem emerges if the momentum integration is confined to the vicinity of the hot spots which in turn requires $\bar{\omega}$ to be smaller than the fermionic bandwidth $W$. This defines a "universal" strong coupling limit: $\lambda \geq 1, \bar{\omega}<W$. The neglect of the $k$-dependence of the $d$-wave gap and of the self-energy along the Fermi surface (i.e., the approximation of $N_{f}$ by a constant) is an $O(1)$ approximation even when $\bar{\omega} \ll W$, but these two $k$-dependences are totally irrelevant from the physics perspective and can be safely omitted [1]. Furthermore, even when $\bar{\omega}>W$, the pairing problem does not change qualitatively except that typical momenta measured from a hot spot are now $O\left(p_{F}\right)$, and $N_{s}$ and $N_{f}$ are no longer universally related. In this regime, the spin part of the condensation energy prevails, and scales as $W^{2} / \bar{\omega} \sim J$, as predicted by Scalapino and White 3.

To conclude, we have shown that at strong spinfermion coupling, the behavior of the condensation energy differs strongly from the BCS prediction. Instead of getting larger as $\Delta$ increases, as predicted by BCS, the total condensation energy flattens off and then decreases. This decrease appears to be correlated to a change in sign of the kinetic energy, another non-BCS type behavior. We also found that the spin and charge contributions to $E_{c}$ are of comparable strength and opposite sign, and negative $E_{c}$ results from a delicate balance between the two. This behavior has no analog for phonon superconductors, where the feedback on bosons is a minor effect.

We thank Ar. Abanov, D. Pines, J. Loram, M. Norman and D. van-der-Marel for useful discussions. This research was supported by NSF DMR-9979749 (A. Ch) and by DR Project 200153, and the Department of Energy, under contract W-7405-ENG-36. (R.H.)
[1] see e.g., J.R. Schrieffer, Theory of Superconductivity, Perseus books, 1999.

[2] J. W. Loram et al, Physica C 235-240, 134 (1994); J. W. Loram et al, Physica C 282-287, 1405 (1997).

[3] D. J. Scalapino and S.R. White, Phys. Rev. B 58, 8222 (1998)

[4] M. R. Norman et al, Phys. Rev. B 61, 14742 (2000).

[5] J. Hirsh and F. Marsiglio, cond-mat/9908322.

[6] A.J. Leggett, Phys. Rev. Lett. 83, 392 (1999).

[7] G.M. Eliashberg, Sov. Phys. JETP 16, 780 (1963).

[8] J.M. Luttinger and J.C. Ward, Phys. Rev. 118, 1418 (1960).

[9] This implies that the chemical potential does not change between the normal and superconducting states. For continuous low-energy theories, this is the case if the theory is free from ultraviolet divergencies. Our computational procedure that involves a transformation to the real axis is ultraviolet convergent.

[10] J. Bardeen and M Stephen, Phys. Rev. B 136, A1485 (1964); see also Y. Wada, Phys. Rev. 135, A1481 (1964). For a recent review, see F. Marsiglio and J.P. Carbotte, cond-mat/0106143.

[11] Ar. Abanov et al, Europhys. Lett., 54, 488 (1999); condmat/0107421: D. Pines et al, cond-mat/0201140.

[12] see e.g., Ar. Abanov et al, cond-mat/0112126. This $N_{f}$ yields $N_{s}=0.17 \mathrm{st} / \mathrm{eV}$ consistent with previous estimates 11]: for $\bar{\omega} \sim 200-250 \mathrm{meV}$ and Fermi surface averaged $v_{F} \sim 0.6 \mathrm{meV}, N_{s}=\left(8 / 3 \pi^{2}\right) \bar{\omega} / v_{F}^{2} \sim 0.15-0.19 s t / e V$.

[13] Ar. Abanov et al, in preparation.

[14] P. Bourges et al., Science 288, 1234 (2000). 